\title{
The rapid evolution of dust content in galaxies over the last five billion years
}

\author{
H. L. Gomez ${ }^{1}$, L. Dunne ${ }^{2}$, D. J. B. Smith ${ }^{3}$, and E. da Cunha ${ }^{4}$ \\ ${ }^{1}$ School of Physics \& Astronomy, Cardiff University, The Parade, Cardiff, CF24 3AA, UK \\ email: haley.gomez@astro.cf.ac.uk \\ ${ }^{2}$ Dept of Physics and Astronomy, University of Canterbury, Private Bag 4800, Christchurch \\ 8140, New Zealand \\ email: loretta.dunne@canterbury.ac.nz \\ ${ }^{3}$ Centre for Astrophysics, Science \& Technology Research Institute, University of \\ Hertfordshire, Hatfield, Herts, AL10 9AB, UK \\ email: smith@herts.ac.uk \\ ${ }^{4}$ Max-Planck Institute for Astronomy, Königstuhl 17, 60115 Heidelberg, Germany \\ email: cunha@mpia-hd.mpg.de
}

\begin{abstract}
The Herschel-ATLAS (H-ATLAS) will provide an unrivalled sample of galaxies, probing the normal star-forming submillimetre population of galaxies for the first time. Here, we exploit the Science Demonstration Phase (SDP) data to model the evolution of the interstellar content of galaxies in recent history. The most massive H-ATLAS galaxies show a large increase in the dust content five billion years ago compared to the present epoch. These observations are difficult to explain using standard dust models, one possibility could be contributions from a non-stellar source of dust e.g. grain growth in dense clouds; this would imply that less than $10 \%$ of dust would be condensed in stellar atmospheres. Alternatively, an initial mass function which becomes top heavy at high star formation rate densities could also explain this discrepancy.
\end{abstract}

Keywords. galaxies: evolution — infrared: galaxies — dust, extinction — submillimeter

\section{Introduction}

After more than two decades of space-based infrared (IR) astronomy, we still know very little about the origin of dust or the build up of heavy elements with time. Herschel (Pilbratt et al. 2010) provides a unique opportunity to resolve this situation, and is an efficient way of tracing the interstellar material in galaxies, and therefore the heavy elements and the fuel required for star formation. Herschel also allows us to survey large areas of the sky and the improved resolution in the submillimetre means we can more reliably identify counterparts to these sources (e.g. Smith et al. 2011). The HerschelATLAS (hereafter H-ATLAS, Eales et al. 2010) is the largest open-time key project on Herschel and has surveyed 550 square degrees of the sky, choosing areas with low cirrus contamination and where (in the equatorial fields), we also have multiwavelength ancillary data from the UV, optical, near-IR through to the submillimetre (submm) thanks to surveys such as GAMA survey (Driver et al. 2011). Here we discuss results from the H-ATLAS SDP data (Fig. 1, left). There are at least 6000 sources in this image with $S / N>5$ (Rigby et al. 2011), of which approximately 2500 have identified optical counterparts in the Sloan Digital Sky Survey (SDSS), with spectroscopic redshifts for 1100 galaxies out to $z<0.5$. 


\section{Dusty H-ATLAS Galaxies}

After extracting sources and identifying their counterparts, the dust mass and other parameters are estimated using the spectral energy distribution (SED), spanning wavelengths from the UV to the submm regime. The SEDs are fit with the MAGPHYS code (da Cunha et al. 2008) which applies an energy-balance technique: the light absorbed by dust in the UV-optical is re-radiated in the IR-submm. Millions of combinations of libraries are used to fit the photometry data, producing a probability distribution function for the resulting parameters including dust mass, temperature and star formation rate (SFR).

So what does the average H-ATLAS galaxy look like? Comparing the dust mass and the SFRs of the SDP sample (Fig. 1, right) suggests the average galaxy (at $z<0.35$ ) has a $\mathrm{SFR}$ of $\sim 3 \mathrm{M}_{\odot} \mathrm{yr}^{-1}$ with a dust-to-stellar mass ratio of 0.004 (Smith et al. 2012). For the same SFR, the galaxies detected by Herschel are dustier than those detected by IRAS as expected since IRAS was more sensitive to the less massive warm dust component due to its shorter wavelength coverage. Compared to the 'traditional' submm galaxies originally detected by SCUBA (typically at higher redshifts), with H-ATLAS we are no longer biased towards detecting the brightest star-forming galaxies but are able to probe the normal submm population for the first time.

In Fig. 2, we compare the dust mass function (DMF) of the H-ATLAS SDP sample in different redshift bins (from Dunne et al. 2011, hereafter D11). For the most massive galaxies, there is a clear evolution in the dust mass which changes by (at least) a factor of five over this redshift range. (Note that the apparent halt in the DMF at $z>0.35$ could be due to the dominance of photometric redshifts compared to spectroscopic redshifts in this bin.) Somewhat surpisingly then, the dust content of the most massive galaxies has decreased by a factor of five in recent history. Although the FIR luminosity function was known to vary over this redshift range, Fig. 2 tells us that this is in part due to the change in dust content rather than only due to a change in dust heating.

We can visualize the rapid decrease in dust content in galaxies by plotting the dust mass density with redshift (D11) as shown in Fig. 2 (right). A similar (but stronger) decrease is also seen in the molecular gas content of Ultra Luminous IR galaxies (ULIRGs) over a similar redshift range (Braun et al. 2011) suggesting the dust decline is closely linked to the gas content. This strong decline in gas and dust may be an natural explanation for the decrease in star-formation rate density (e.g. Hopkins 2004).
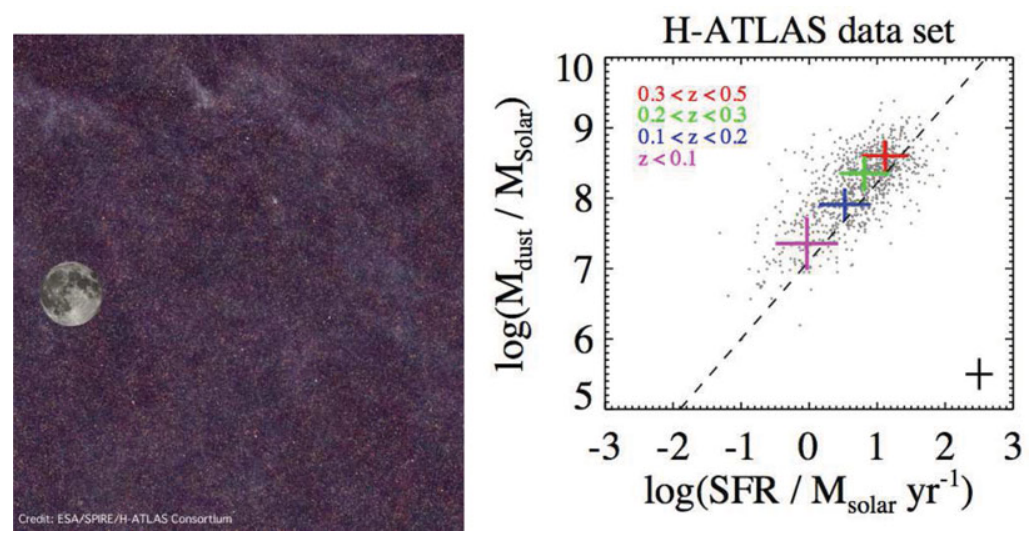

Figure 1. Left: The three-colour SPIRE H-ATLAS SDP image with the Moon shown to scale. Right: $M_{d}$ versus star formation rate (from Smith et al. 2012), crosses show the average values. Overplotted is the best fit relationship from da Cunha et al. 2010. 

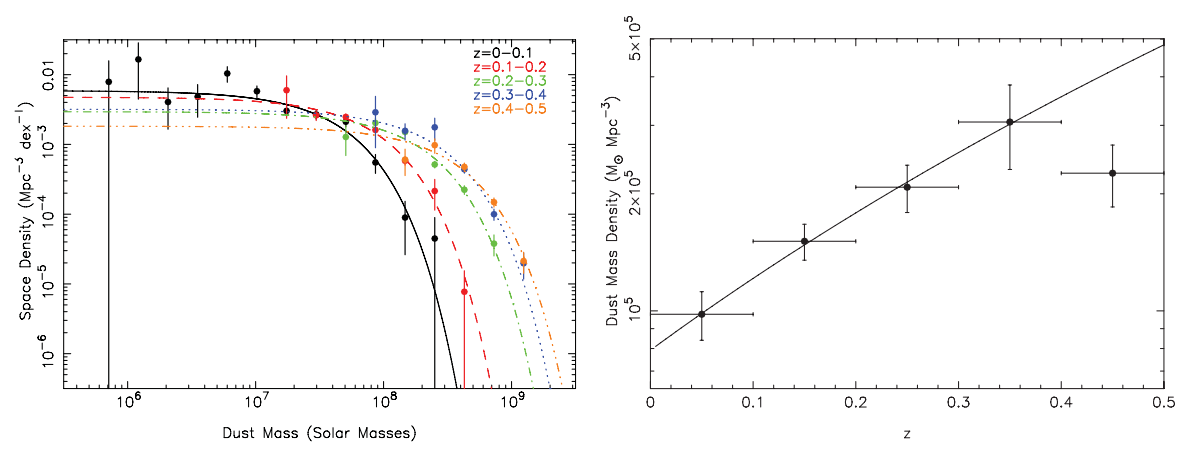

Figure 2. Left: The dust mass function of the H-ATLAS galaxies in the SDP field (from D11). Right: The dust mass density $\rho_{d}$ versus redshift. The solid curve is the relationship $\rho_{d} \propto(1+z)^{4.5}$.

\section{Modelling the Dust Life-Cycle}

Here, we use a simple chemical evolution model to investigate the decrease in dust content of galaxies. The model follows the metal and dust yields from stars over time as star formation depletes the gas (D11) and assumes approximately $20 \%$ of the metals ejected by core-collapse supernovae (SN) condense into dust (e.g. Todini \& Ferrara 2001; Gomez et al. submitted). Theoretical modelling of AGB stars suggest intermediate mass stars convert $20-40 \%$ of their ejected metals into dust (e.g. Morgan \& Edmunds 2003).

If we compare the results from the chemical evolution model with the H-ATLAS observations we find two problems. In Fig. 3 (left) we compare the dust mass versus the SFR of the H-ATLAS sample, with three possible stellar dust contributions: (i) AGB stardust only (ii) stardust and SN dust and (iii) conversion of all of the SN metals into dust. Fig. 3 highlights that SNe must contribute to the total dust budget in galaxies. However, to account for the dust masses of the H-ATLAS sample, we would require each core-collapse SN to inject a net dust mass of $1 \mathrm{M}_{\odot}$ into the ISM. Given the harsh environment of SN shocks, this seems unphysical. The large dust masses detected by H-ATLAS may suggest that another source of dust is needed, one in which 'free' metals in the ISM can condense into dust grains. One mechanism could be grain growth in molecular clouds, though this would require that $90 \%$ of the dust mass in galaxies was actually formed in the ISM and not in stellar atmospheres (e.g. Draine 2009). Alternatively, the dust yield from the model would be significantly increased if the Initial Mass Function (IMF) determining the number of stars of a given mass formed in a stellar population is 'heavier' than the standard universal Salpeter/Scalo form. An IMF of the form $\phi(m) \propto m^{-1.7}$ would certainly increase the metals from a stellar population and hence the dust mass by a factor of four, easily accounting for the highest $M_{\mathrm{d}} / M_{*}$ ratios seen by Herschel.

It is also extremely difficult to account for the rapid evolution in the dust content during the last five billion years. Fig. 3 (right) compares the observed decrease in $M_{d} / M_{*}$ over time with the model. Grain growth in the ISM increases the dust mass, but to reproduce the evolution over time, a change in the IMF is required. Specifically, an IMF which is top heavy at high SFR densities (e.g. Papadopoulos et al. 2011) reproduces this trend.

\section{Discussion}

Using Herschel, we have derived the dust properties of $\sim 1400$ galaxies. We find that:

- H-ATLAS galaxies at $z \leqslant 0.5$ are dustier than previous samples detected by IRAS.

- The evolution seen in the DMF corresponds to a factor 4-5 increase in the dust masses 

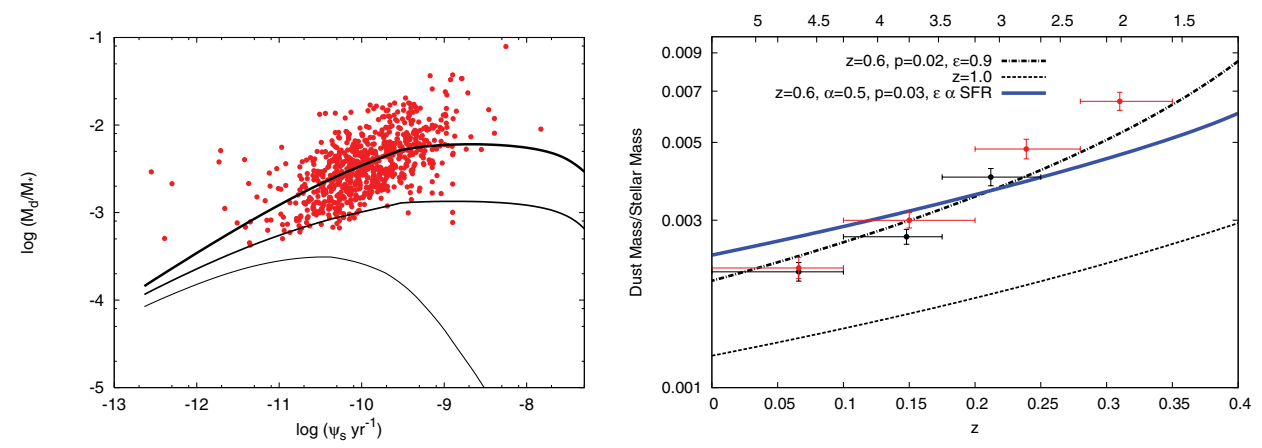

Figure 3. Left: $M_{d}$ versus specific SFR $\psi_{\mathrm{s}}$, with a chemical evolution model (Gomez et al. in prep.). From top to bottom, curves show (a) dust from $\mathrm{SNe}(100 \%$ efficiency) and stars (b) dust from SNe (20\% efficiency) and stars and (c) no SN contribution to dust. Right: The change in $M_{d} / M_{*}$ with $z$ (from D11). Black points are sources with spectroscopic $z$ (red includes photometric $z$ ). The Milky Way model (Morgan et al. 2003) is the dashed curve. To fit the data, we require (i) $90 \%$ of dust to be formed via grain growth in the ISM ( $\epsilon$, dot-dashed) or (ii) a total yield of $p>0.02$ (a top-heavy IMF) combined with some grain growth in the ISM (blue).

of the most massive galaxies in recent cosmic history. This is suggestive of galaxies being more gas rich at $z=0.5$.

- To account for the evolution of the dust content we need to radically alter chemical and dust evolution models. We cannot reproduce these trends with Milky Way yields.

- We suggest that the H-ATLAS galaxies are more efficient at converting metals into dust through grain growth in the ISM (which would then account for $90 \%$ of the dust mass in galaxies). Alternatively, higher metal yields from stars can be achieved through an IMF which becomes top-heavy at high SFR densities.

This dataset is only $3 \%$ of the entire areal coverage of the H-ATLAS, with hundreds of thousands of sources now detected by Herschel. Perhaps the biggest limitation now to our understanding of the submm population is the urgent need for reliable counterparts and spectroscopic redshifts of these sources.

\section{Acknowledgments}

We acknowledge the H-ATLAS consortium (http://www.h-atlas.org) and GAMA.

\section{References}

Braun R., Popping A., Brooks K., \& Combes F., 2011, MNRAS, 416, 2600

da Cunha E., Charlot, S., \& Elbaz D., 2008 MNRAS, 388, 1595

da Cunha E., Eminian C., Charlot S., \& Blaizot J., 2010, MNRAS, 403, 1894

Draine B. T., 2009, in Cosmic Dust - Near and Far, ASP, Vol. 414, p.453

Driver S. P., Hill D. T., Kelvin L. S., et al. 2011, MNRAS, 413, 971

Dunne L., Gomez H. L., da Cunha E., et al. 2011, MNRAS, 417, 1510, D11

Eales S. A., Dunne L., Clements D., et al. 2010, PASP, 122, 499

Hopkins, A. M., 2004, ApJ, 615, 209

Morgan H. L. \& Edmunds M. G., 2003, MNRAS, 343, 427

Papadopoulos P. P., Thi W.-F., Miniati F., \& Viti S., 2011, MNRAS, 414, 1705

Pilbratt G. L., Riedinger J. R., Passvogel T., et al. 2010, A \& A, 518, L1

Rigby E. E., Maddox S. J., \& Dunne L., MNRAS, 415, 2336

Smith D. J. B., Dunne L., Maddox S., et al. 2011, MNRAS, 416, 857

Smith D. J. B., Dunne L., da Cunha E., et al. 2012b, MNRAS, in press, arXiv: 1208.3079

Todini P. \& Ferrara A., 2001, MNRAS, 325, 726 